\title{
The citizen-candidate model with imperfect policy control: Strategic delegation and polarization
}

\author{
R. Emre Aytimur Chair of Public Economics, Georg-August University \\ Göttingen \\ Aristotelis Boukouras Department of Economics, University of Leicester \\ Robert Schwager Chair of Public Economics, Georg-August University \\ Göttingen
}

\begin{abstract}
We present a modified citizen-candidate model where the implemented policy arises from a compromise between the government and an unelected external power. We show that the two-candidate equilibria of this model differ significantly from the original: however small the cost of candidacy, the distance between the candidates' policies, both ideal and implemented, remains strictly above a threshold. Moreover, there may be one-candidate equilibria in which the only candidate is not the one most preferred by the median voter. Both results point out that, even with negligible cost of entry, there are limits to strategic delegation.
\end{abstract}

JEL classification: D72, D78

Corresponding author: Robert Schwager, rschwag@uni-goettingen.de

\section{Introduction}

The recent economic crisis within the Eurozone has revealed an interesting aspect of political economics. Many Southern European governments need to implement policies which are opposed by a large proportion of voters, and voters seem to be driven to the extremes as a result. Indeed, in the case of Greece, the recent political turmoil led to a dispersion of voters among multiple parties in the latest election which took place in January 2015. Specifically, after the center-right Nea Demokratia and the center-left Pasok had been the dominating forces of Greek politics for three decades, now Syriza, a party located much farther to the left, has ousted both parties from the centre stage and has taken control of the government. Thus, as a result of the crisis, the ideological distance between Syriza on the left and Nea Demokratia on the right has increased substantially. Moreover, the popular support that Syriza enjoyed in its first few months as a ruling party makes clear that the majority of Greek population would prefer much less austere policy measures, or, put differently, the implemented policies differ substantially from the ideal policy of the median 
voter. How can this increased distance between the political positions of the major contenders, and the divergence between the preferred policy of the median voter and the implemented policy be reconciled by the traditional public choice framework?

We answer this question by using the celebrated citizen-candidate model, which was pioneered by Osborne and Slivinsky (1996) and Besley and Coate (1997). In two-candidate equilibria of this model, a substantial cost of running for office prevents a convergence of policy platforms. Conversely, if the cost of candidacy is small, the model allows for equilibria with two candidates proposing policies which are arbitrarily close to each other and to the median's preferred policy (see Persson and Tabellini 2002, p. 101-104). In the present paper, we show that this latter conclusion is no longer true if the elected government cannot fully control the policy to be implemented but has to compromise with an external power. If the final policy is in between the ideal policies of the elected citizen and the external power, then in any two-candidate equilibrium the distances between the ideal policies and between the policies finally implemented by the two candidates remain strictly above a positive threshold, even when the cost of running for office becomes arbitrarily small.

As our earlier example indicates, the political importance of this result stems from the observation that quite often, elected governments have to share power with an unelected entity. For example, a self-interested bureaucracy in the spirit of Niskanen (1971) may, by its expertise or its control on executive functions, 'water down' implemented policies. Similarly, an interest group ${ }^{1}$ which has the means to disrupt public life, such as a union or an industry association, can influence the policies effectively enacted by the government. As a third example, in developing countries, even elected governments often feel compelled to take the views of donor countries into account when formulating domestic policies. Finally, as we have already pointed out, when the International Monetary Fund or, recently, the European Union negotiate economic programs with countries receiving debt relief, the resulting policies clearly arise as a compromise between the preferences of the elected government and those of the international institution. For these and similar situations, our result implies that a polarization of candidates and policies is inevitable.

To arrive at this conclusion, we present a simple model where citizens have single-peaked preferences over an unbounded one-dimensional policy space, decide non-cooperatively whether to stand in an election, and vote strategically for one of the candidates. To formalize the influence of the external power, we assume that the final policy is a weighted average of the winner's ideal policy and the external power's preferred choice. As our main result, we show that the ideal policies of two candidates running in an equilibrium, and also the policies finally implemented in case of victory, must differ by a minimum amount. This minimal distance increases in the strength of the external power and in the difference between the median voter's and its preferred policies, but is independent of the cost of candidacy. This result obtains since otherwise, if two candidates with similar preferences were to run, one of them would prefer the compromise between the other candidate and the external power to the compromise she can obtain herself.

While two-candidate equilibria are arguably more realistic than uncontested elections, for the sake of completeness, we study as well one-candidate equilibria. We find that these equilibria also differ in an interesting way from those of the standard model. In the standard model, the median voter being the candidate is the only one-candidate equilibrium for a sufficiently small cost of

\footnotetext{
${ }^{1}$ Besley and Coate (2001) study the influence of lobbying in a citizen-candidate model. However, since the government has full control over the policy in their model, they do not obtain our result.
} 
entry. In our model, under a mild assumption, the analogue of this equilibrium exists, i.e. there is a one-candidate equilibrium in which the citizen who runs for office implements a policy which is preferred by the median to all policy compromises that could be achieved by any other candidate. However, even when the cost of running for office is arbitrarily small, there remain other onecandidate equilibria in which the implemented policy is substantially different from the median voter's preferred one.

Our analysis is in line with several other contributions which show that adding institutional features to the standard citizen-candidate model can change the candidates' policies observed in an equilibrium. In a model serving to analyze empirically the reservation of elected office to women in India, Chattopadhyay and Duflo (2004) consider a policy-formation rule similar to the one presented here. They focus on the incentives of women to stand for office, however, and do not address the issue of policy divergence. Chambers (2007) provides a model where lobbies pay campaign contributions to potential candidates so as to convince them to run. He shows that this induces a minimum distance between the policies chosen in two-candidate equilibria. Our approach differs from this result in that we consider an external power which influences the policy after the election, rather than manipulating the election itself. It has also been shown that ideal policies in a two-candidate equilibrium must be sufficiently far apart if the final policy is a weighted average of the ideal policies of all candidates (Hamlin and Hjortlund 2000), or if the decision to stand in the election must be taken before the distribution of voter preferences is known (Brusco and Roy 2011). Both Hamlin and Hjortlund (2000) and Brusco and Roy (2011) assume sincere voting, and the driving force behind the divergence results is the threat of a third candidate entering on the fringe of the political spectrum. Contrary to that, in our model, which is based on strategic voting, it does not pay off for the second candidate to enter if policies are too close to each other. Thus, while these contributions arrive at similar conclusions, our result is based on a fundamentally different effect.

Our results also contribute to the theory of strategic delegation in a political context. This strand of literature emphasizes that the median voter, by electing someone with preferences different from herself, can compensate for unwelcome influences in the post-election decision making, and thereby implement her preferred policy. For example, Persson and Tabellini (1992) show that electing a citizen who likes higher taxes than the median is a way to counteract the race to the bottom endemic in tax competition. Similarly, Roelfsema (2007) shows that strategic delegation can overcome the free-riding incentives present when countries set environmental standards in an uncoordinated way. In an intertemporal setup, electing a citizen with a high endowment of capital is a way to commit to a low tax rate on capital, thereby preserving incentives to invest (Persson and Tabellini 1994). Other applications of strategic delegation refer, for example, to joint production of a public good (Harstad 2010), to monetary policy in the European Central Bank (Fatum 2006), or even to civil conflicts (Jennings and Roelfsema 2008).

In comparison, our paper yields results which go to the opposite direction. Specifically, in any two-candidate equilibrium of the model, at least one of the final policies remains bounded away from the median's ideal policy, even for arbitrarily small cost of running for office. The one-candidate equilibria of our model produce a similar result. Even though, provided the cost of candidacy is not too high, there is an equilibrium where the only candidate is the median's preferred choice, this equilibrium is not unique. In other words, strategic delegation may not work even for one-candidate equilibria and even for an arbitrarily small cost of entry. Thus, our results show that the power of strategic delegation is limited, in particular as long as one considers equilibria where 
elections are indeed contested.

The rest of the paper is organized as follows. Section 2 presents the model. Section 3 analyzes both two- and one-candidate equilibria. Whereas subsection 3.1 presents the general case, subsection 3.2 illustrates the results further through a more specific case with linear symmetric utility. Section 4 shortly discusses the results and concludes. Many proofs are in the appendix.

\section{The Model}

There are $n$ citizens with $n$ odd. $N$ denotes the set of citizens. Each citizen has preferences over a unidimensional policy $p \in \mathbb{R}$ represented by the utility function $u_{i}(p)$. The ideal policy point of a citizen $i$ is denoted by $p_{i}$. For the sake of concreteness, we say that policy $p$ is to the left (right) of policy $q$ if $p<q(p>q)$. In the same way, we label citizen $i$ as being more left(right-) wing than citizen $j$ if $p_{i}<p_{j}\left(p_{i}>p_{j}\right)$. The median voter's ideal policy is denoted by $p_{m}$. In general, we only assume that preferences are single-peaked. For purposes of illustration, however, in subsection 3.2, we also consider the special case where utility functions are linear and symmetric, i.e., $u_{i}(p)=-\left|p-p_{i}\right|$ for all $i$.

There are three stages. In the first stage, each citizen decides whether to stand for election or not. Being a candidate costs $c>0$. In the second stage, voting takes place according to the plurality rule. In case of a tie, every candidate who ties for the first place is selected as the winner with equal probability. In the third stage, if citizen $i$ is the winner of the election, then the final policy $p_{i x}$ is a weighted average of her ideal policy $\left(p_{i}\right)$ and the ideal policy of an external power $\left(p_{x}\right)$ :

$$
p_{i x}=\gamma p_{i}+(1-\gamma) p_{x}
$$

with $0<\gamma \leq 1$. This formula captures the idea that, ideally, the election's winner would like to implement her most preferred policy, but has to compromise with the external power. The expression $1-\gamma$ measures the power of this unelected entity. ${ }^{2}$ If no one runs for the election, then the final policy becomes $p_{x}$. We assume without loss of generality that $p_{m}<p_{x}$, i.e. the outside power prefers a more right-wing policy than the majority of the electorate.

The equilibrium concept is subgame perfect equilibrium together with the elimination of weakly dominated voting strategies. Our focus is on equilibria with one or two candidates standing in the election, but we will briefly comment on equilibria with three or more candidates at the end of subsection 3.1.

\section{Results}

The analysis starts in subsection 3.1 with the general model, where single-peakedness is assumed throughout. In subsection 3.2 we illustrate the main findings in the special model with linear utility functions. In both subsections we start by analyzing equilibria with two candidates, and then turn to one-candidate equilibria.

\footnotetext{
${ }^{2}$ In (1), $p_{i}$ should not be misunderstood as a policy which the elected government could choose freely, since then the outside influence could simply be undone by a choice which implements $p_{i}$. Rather, $p_{i}$ represents the preferences of the government, and (1) describes the outcome of some form of bargaining between the government and the external power. Clearly, for the idea of imperfect policy control to make sense, this must in general diverge from the government's ideal policy.
} 


\subsection{Single-peaked Preferences}

From Proposition 3 in Besley and Coate (1997) we know that a two-candidate equilibrium exists only if (a) the number of citizens who strictly prefer one candidate to the other is the same for both candidates, and (b) for both candidates, the gain in expected utility arising from changing the final policy with probability $1 / 2$ outweighs the cost of running. ${ }^{3}$ Condition (a) requires that the two candidates tie in an election since otherwise, the candidate who is bound to lose would pay the cost $c>0$ without changing the outcome. Condition (b) implies that each candidate values her impact on the expected outcome more than the cost of running.

Different from Besley and Coate (1997), in our model, voters and candidates evaluate final policies $p_{i x}$ and $p_{j x}$ rather than candidates' ideal policies $p_{i}$ and $p_{j}$ when deciding for whom to vote and whether to run. This is important because it leads to a minimum distance between the ideal policies of candidates in two-candidate equilibria. The divergence in candidate's positions gives rise to a divergence of equilibrium implemented policies, which persists even when the cost of entry is arbitrarily small. We formalize this result in the proposition below, where we make use of necessary conditions (a) and (b) and equation (1).

PROPOSITION 1. In any equilibrium with two candidates $i$ and $j,\left|p_{i}-p_{j}\right|>[(1-\gamma) / \gamma]\left(p_{x}-p_{m}\right)$.

Proof. Note that $p_{i} \neq p_{j}$, since otherwise one of the candidates would be better off not running for the election and saving the $\operatorname{cost} c$, violating condition (b). Assume without loss of generality that $p_{j}=p_{i}+d$ with $d>0$. With (1), this implies $p_{j x}>p_{i x}$. The key observation is that $p_{j}>p_{i x}$, since otherwise $j$ would prefer $p_{i x}$ to $p_{j x}$ (due to single-peaked preferences) and would be certainly better off not running for the election (even with $c=0$ ), again contradicting condition (b). This can be equivalently written as $p_{i}+d>\gamma p_{i}+(1-\gamma) p_{x}$ which gives

$$
d>(1-\gamma)\left(p_{x}-p_{i}\right)
$$

From condition (a), in a two-candidate equilibrium, the candidates should tie. Due to singlepeaked preferences, this is possible only if $p_{j x}>p_{m}>p_{i x}$. By substituting equation (1) for $p_{i x}$ into $p_{m}>p_{i x}$ and rearranging, we get: $p_{x}-p_{i}>\left(p_{x}-p_{m}\right) / \gamma$. Combining this with inequality (2) gives

$$
d>\frac{1-\gamma}{\gamma}\left(p_{x}-p_{m}\right)
$$

QED

Proposition 1 says that it is not possible to have two candidates whose ideal policies are at a

\footnotetext{
${ }^{3}$ Besley and Coate (1997) also show that the necessary conditions (a) and (b) together are sufficient for existence of a two-candidate equilibrium if in addition, (c) the number of voters who are indifferent between the two candidates is smaller than one third of the electorate minus 1. Condition (c) makes sure that a third candidate does not enter, both in the standard and in our model, which share the same structure at the voting stage. If a third candidate were to enter, every voter not indifferent between the two candidates would not change their votes, thinking that the third candidate is not a serious contender (which is confirmed in equilibrium given condition (c)). Indifferent voters between two candidates may switch their vote to the third candidate, but if condition (c) is satisfied, it would still be true that the third candidate has no chance of winning and so would not enter the race.
} 
distance lower than $[(1-\gamma) / \gamma]\left(p_{x}-p_{m}\right)$ from each other, even if the cost of running as a candidate is arbitrarily small. The next corollary, which follows directly from Proposition 1 and equation (1), shows that correspondingly, the two potential final policies are distant from each other by at least $(1-\gamma)\left(p_{x}-p_{m}\right)$.

COROLLARY 1. In any equilibrium with two candidates $i$ and $j,\left|p_{i x}-p_{j x}\right|>(1-\gamma)\left(p_{x}-p_{m}\right)$.

Corollary 1 highlights the difference between our model and the standard citizen-candidate model. Since in a two-candidate equilibrium the median's preferred policy must be located between the policies implemented by the two potential winners, that model provides an institutional framework for (almost) implementing the median's preferences. Contrary to that, when an external power influences the policy outcome, at least one of the final policies necessarily stays bounded away from the median preferred policy, no matter how small the cost of running is.

In addition, from Proposition 1 and Corollary 1 one sees that naturally, as the bargaining power $1-\gamma$ of the external influence increases, or as the ideological distance $p_{x}-p_{m}$ between the median voter and the external power increases, our model diverges more from the standard model, and results in a higher minimum distance between candidates' platforms and final policies.

To understand the effect driving these results, we discuss different possible equilibrium constellations. We remind the reader that we consider, without loss of generality, the case where the median's preferred policy is located to the left of the policy preferred by the outside force, so that $p_{m}<p_{x}$. One possible equilibrium constellation consists in having a left-wing candidate $i$ and a right-wing candidate $j$ whose ideal policies are positioned on different sides of $p_{x}$, i.e. $p_{i}<p_{x}<p_{j}$. In such a situation, the final policy $p_{j x}$ implemented by the right-wing candidate is to the right of $p_{x}$. Since the median must be indifferent between both candidates, the final policy $p_{i x}$ achieved by the left-wing candidate must then be to the left of $p_{m}$. Therefore, the length of the interval $\left(p_{m}, p_{x}\right)$ provides a lower bound for the distance between final policies in such an equilibrium, which translates into an even larger distance between ideal points of candidates.

There may be a second type of equilibrium, however, where the ideal points of candidates, just like the median's, are also both located to the left of $p_{x}$. Note that this is the most interesting case where imperfect policy control is felt very strongly, since the majority of the population and the political contenders all agree that the outside force's policy prescription should be shifted towards the left. With negligible cost of running for office, one might now expect that an equilibrium with any such pair of candidates is possible, provided they implement final policies which are equally good from the median's point of view. However, if the candidates' ideal points are similar, the candidate whose ideal policy is closer to $p_{x}$ (the "right-wing" $j$ ), would like to lose an election against her opponent (the "left-wing" $i$ ), since the left-wing candidate pulls the final policy more strongly away from $p_{x}$ in the direction desired by both of them. Only when the left-wing becomes so extreme that she pulls the final policy beyond the ideal policy of the right-wing does it become worthwhile for the right-wing to run against $i$.

The present model reduces to the standard citizen-candidate model with perfect policy control when we set $\gamma=1$ in (1). Clearly, then, the inequalities in Proposition 1 and Corollary 1 become trivial. Therefore, there may well be an equilibrium with two candidates whose ideal points are arbitrarily close to each other, provided the cost of running is sufficiently small. ${ }^{4}$ A similar result

\footnotetext{
${ }^{4}$ For instance, if voter $m$ is indifferent between $p_{i}$ and $p_{j}$ and half of the remaining $n-3$ voters respectively have
} 
holds when the external power's ideal policy $p_{x}$ approaches $p_{m}$, even though the limit model with $p_{x} \rightarrow p_{m}$ is not identical to Besley and Coate (1997), since implemented policies still differ from ideal policy positions. Intuitively, when the external power's ideal policy coincides with the median voter's, the implemented positions of both left-wing and right-wing candidates are pulled toward the center, rather than to the right. Then, equilibrium candidates must be positioned on different sides of $p_{x}$, and hence each one of them prefers her own final policy to the one procured by the opponent, however close their ideal points may be. Therefore, with $p_{x} \rightarrow p_{m}$ both have an incentive to run if the cost of entry is low, and the minimum distance of the candidates becomes arbitrarily small.

We now turn our attention to the examination of the one-candidate equilibria. Our results on this type of equilibrium set are similar to the results provided by Besley and Coate (1997) to some extent, but they also indicate some key differences. More specifically, we know from Besley and Coate (1997) that the necessary and sufficient conditions for the existence of a one-candidate equilibrium are (a) that the candidate should prefer to run in the election rather than not run and (b) that any opponent who might tie with or win over the candidate must not find it profitable to run for the election. These two conditions are also necessary and sufficient in our model. However, the set of candidates who satisfy these conditions may differ substantially from Besley and Coate (1997).

In Besley and Coate (1997), the median voter running for office and implementing her most preferred policy is always a one-candidate equilibrium, provided that $c$ is not too high. The corresponding equilibrium in our model would be the citizen whose final policy would be the most preferred by the median voter running for office as the only candidate. To make this idea precise, we define the set $N_{m}^{*}=\left\{i \in N \mid u_{m}\left(p_{i x}\right) \geq u_{m}\left(p_{j x}\right)\right.$ for all $\left.j \in N\right\}$ of citizens who yield the highest utility for the median. If a citizen from this set running for office constitutes a one-candidate equilibrium, we can at least say that there is a one-candidate equilibrium in which strategic delegation works in the sense of resulting in the preferred outcome of the median voter. Surprisingly, this need not be the case. This is because the implemented policy of such a citizen, say $i$, does not necessarily coincide with the median voter's position $p_{m}$, implying that citizen $i$ cannot be sure to win a majority in every race. If, for example, $p_{i x}<p_{m}$ and the opponent candidate is to the right of $i$, the citizens with ideal points in-between $p_{i x}$ and $p_{m}$ may prefer the more right-wing opponent even though they themselves are more left-wing than the median voter, which may result in $i$ losing the election.

Nonetheless, this result requires that the policy preferences of the median voter are substantially different from the preferences of the citizens close to her, which seems an unlikely possibility. To rule it out, we invoke the well-known single-crossing condition (Persson and Tabellini 2002, p. 23):

A1 Consider two final policies $p$ and $q$ and two citizens $i, j$ with ideal policies $p_{i}$ and $p_{j}$. If $q>p$ and $p_{j}>p_{i}$, or if $q<p$ and $p_{j}<p_{i}$, then $u_{i}(q) \geq u_{i}(p) \Rightarrow u_{j}(q)>u_{j}(p)$.

This condition states that if a citizen prefers a left-wing policy to a right-wing policy then citizens to her left also prefer the left-wing policy over the right-wing policy, and vice versa.

With condition A1, the median voter is decisive for election outcomes. For example, when in a vote among two contenders, the median voter strictly prefers the more left-wing candidate, then

ideal policies smaller than $p_{i}$ and larger than $p_{j}$, then a two-candidate equilibrium with $i$ and $j$ running exists whenever the cost of running is small enough. 
A1 implies that all voters whose ideal policy is to the left of the median's will also vote for the left candidate, which will result in the victory of the left candidate.

If preferences satisfy single crossing, then there is always a one-candidate equilibrium in which a citizen most preferred by the median voter is the only candidate. Before presenting this result formally in the following Proposition 2, we need another definition: Since there may be two citizens $i, j \in N_{m}^{*}$ with different ideal policies, yielding the same utility for the median voter, $u_{m}\left(p_{i x}\right)=u_{m}\left(p_{j x}\right)$, we define $p_{i^{*}}=\min \left\{p_{i} \mid i \in N_{m}^{*}\right\}$ and denote by $i^{*}$ any citizen with such an ideal policy. Thus, $i^{*}$ is the median's most preferred candidate, and if there are several optimal candidates, it is the most left-wing among them.

PROPOSITION 2. If $u_{i^{*}}\left(p_{i^{*}}\right)-u_{i^{*}}\left(p_{x}\right) \geq c$ and assumption Al holds, then there exists a onecandidate equilibrium in which $i^{*}$ runs unopposed.

Proof. In the appendix.

It should be noted that the single crossing condition A1 is far stronger than what is required for Proposition 2. While the condition restricts the preferences of any two citizens regarding any two policies, all we need is that citizens with ideal policies between $p_{i^{*} x}$ and $p_{m}$ agree with the median that $p_{i^{*} x}$ is the best possible implementable policy. Then, $i^{*}$ running for office is always a one candidate equilibrium.

In the standard model, we know that for sufficiently small values of $c$, there is a unique onecandidate equilibrium, and that the median voter runs for office in this equilibrium. The next interesting question then is whether there also exist one-candidate equilibria in which the candidate is not a most preferred citizen by the median voter even for arbitrarily small values of $c$. This would mean an instance of failure of strategic delegation. To answer this question together with a full characterization of the set of one-candidate equilibria, we need to admit, in addition to single crossing, the following mild restriction on the citizens' utility functions:

A2 For any $i, j \in N, u_{j}\left(p_{i x}\right) \geq u_{j}\left(p_{j x}\right) \Rightarrow u_{k}\left(p_{i x}\right) \geq u_{k}\left(p_{k x}\right)$ for all $k \in N$ with $p_{k} \in$ $\left[\min \left\{p_{i} ; p_{j}\right\}, \max \left\{p_{i} ; p_{j}\right\}\right]$.

Assumption A2 states that if a citizen $j$ prefers not to run against some candidate $i$ because she prefers that candidate's final policy $p_{i x}$ to her own implemented policy $p_{j x}$, then any citizen $k$ with ideal policy between the two prefers not to run against candidate $i$ either. In other words, assumption A1 puts restrictions on how much voting preferences for neighboring citizens can vary, and assumption A2 extends this reasoning to the entry decision of candidates. ${ }^{5}$

Assumptions A1 and A2 along with the single-peakedness condition are sufficient for a full characterization of the one-candidate equilibria when $c$ is arbitrarily small. This is provided in the following Proposition 3. In this proposition, $p_{l}=\min \left\{p_{i} \mid u_{m}\left(p_{i x}\right) \geq u_{m}\left(p_{m x}\right), i \in N\right\}$ is defined to be the ideal policy of the most left-wing candidate $l$ among those whom the median voter prefers to herself.

\footnotetext{
${ }^{5}$ One can show that assumptions A1 and A2 are satisfied if all voters have symmetric utility functions, which need not necessarily be identical. The formal proof is available from the authors upon request. We are thankful to the editor for suggesting this result to us.
} 
PROPOSITION 3. If Assumptions A1 and A2 hold, for a sufficiently small cost of entry c, a citizen $i$ running for office unopposed constitutes a one-candidate equilibrium if and only if $p_{i} \in\left[p_{l}, p_{i^{*}}\right]$.

Proof. In the appendix.

The above results point toward the same direction as the result on the two candidate equilibria: under single-peaked preferences, a model where the implemented policy is a result of a compromise with an external power may lead to an equilibrium policy other than the one most preferred by the median voter. Even when one adds additional assumptions to utility functions, such as A1 and A2, in order to guarantee that the median's most preferred policy is a one-candidate equilibrium policy, it may not be unique. As opposed to the standard model, other political equilibria are possible even for arbitrarily small costs of entry, some of which may involve final policies which are quite far away from the ideal point of the median voter.

These additional equilibrium outcomes are supported by the same strategic consideration which rules out two-candidate equilibria where both contenders have similar ideal points. A possible second candidate may actually prefer the compromise which the citizen already standing for election will reach with the outside power to what she can achieve herself. Therefore, even with low cost of running for office, a candidate who will implement a policy at some distance from the median voter's preferred policy will be protected from entry by a competitor. Altogether, this implies that in a one-candidate equilibrium, the outside power induces the same strategic considerations as in a two-candidate equilibrium, but this has less severe consequences: In the one-candidate case, an equilibrium where the median voter's preferred outcome occurs is still possible, whereas with two candidates, at least one of the candidates must be rather extreme.

In one-candidate equilibria where strategic delegation fails, i.e., where the only candidate $i$ is not the candidate $i^{*}$ preferred by the median, the final policy is always to the left of the policy which $i^{*}$ would have implemented. To understand why, note that any such candidate $i$ would lose against $i^{*}$ should $i^{*}$ decide to run. When the candidate $i$ is to the right of $i^{*}$, this will indeed happen since by winning the election, $i^{*}$ can pull the final policy further to the left and hence improve her utility. In contrast, when the candidate $i$ implements a policy somewhat to the left of the final policy provided by $i^{*}$, citizen $i^{*}$ prefers $i$ to herself and therefore refrains from entering the race. Assumption A2 guarantees that this reasoning extends to all citizens with preferences similar to $i^{*}$, so that candidate $i$ is protected against entry by any opponent.

Finally, with respect to this class of equilibria, note that as $p_{x}$ approaches $p_{m}$, both $p_{l}$ and $p_{i^{*}}$ approach $p_{m}$ so that the equilibrium set in Proposition 3 collapses to a single point, i.e. the median voter's position. Intuitively, when the outside power has almost the same preferences as the median, there is no candidate whom the median prefers to herself, and so any other candidate would be challenged by the median. In this sense, the limit case where $p_{x} \rightarrow p_{m}$ reproduces the median voter result known from the standard citizen-candidate model.

The game has also equilibria with more than two candidates, which is a general feature of citizencandidate models. Our main result about two-candidate equilibria giving a minimum distance between the candidates is not generalizable to those equilibria. To understand why, observe that in the case of two-candidate equilibria, each candidate has to prefer her final policy over the other candidate's, and recall that Proposition 1 rests on the fact that this property cannot be satisfied 
if candidates' ideal policies are too close. In contrast, in case of three or more candidates, this property is not necessary for an equilibrium any more. For instance, even if a candidate likes her final policy the least, she can still run if she believes the electoral outcome is going to be worse in case of her withdrawal. This is the reason why there may exist equilibria with more than two arbitrarily close candidates. ${ }^{6}$ Consequently, following established practice in the literature as well (see for example, Besley and Coate (2001) and Chattopadhyay and Duflo (2004)), we omit a further analysis of these equilibria.

We now turn to illustrating the general results by means of the special model with linear utility functions. Moreover, for this specification we can completely characterize the set of two-candidate equilibria for any cost of running $c>0$, and compare these with equilibria obtained in the standard citizen-candidate model with perfect policy control.

\subsection{Linear Symmetric Utility}

In this subsection, preferences of all agents $i$ are described by the utility function $u_{i}(p)=-\left|p-p_{i}\right|$. Note that this linear symmetric specification of the utility function satisfies assumptions A1 and A2 of subsection 3.1. In the case of two candidates, we call them $i$ and $j$, and assume without loss of generality that $p_{i}<p_{j}$. We refer to $i$ as the left-wing candidate and $j$ as the right-wing candidate.

We assume throughout this section that the number of voters with ideal policy $p_{m}$ falls short of one third of the electorate by more than one voter. In the case of linear symmetric utilities, with this assumption, the necessary conditions (a) and (b) stated in subsection 3.1 are also sufficient for a two-candidate equilibrium (see footnote 3 ).

We characterize the set of two-candidate equilibria by the following proposition:

PROPOSITION 4. Assume that utility functions are given by $u_{i}(p)=-\left|p-p_{i}\right|$ for all $i$ and that the number of citizens with the median ideal policy $p_{m}$ is less than $\frac{n}{3}-1$. Then, there exists a two-candidate equilibrium in which citizens $i$ and $j$ run against each other if and only if

(a) $\frac{p_{i x}+p_{j x}}{2}=p_{m}$, and

(b) $p_{j x}-p_{i x} \geq 2(1-\gamma)\left(p_{x}-p_{m}\right)+2 \gamma c$ for $c<p_{x}-p_{m}$; and $p_{j x}-p_{i x} \geq 2 c$ for $c \geq p_{x}-p_{m}$.

Proof. In the appendix.

Proposition 7 by Besley and Coate (1997) characterizes two-candidate equilibria of the standard model with the the same linear symmetric utility function $u_{i}(p)=-\left|p-p_{i}\right|$. That proposition can be obtained from our Proposition 4 as a special case by setting $\gamma=1$.

The differences between the standard model and our model with imperfect policy control are illustrated in Figures 1 and 2. The first figure depicts the minimum distance between final policies as a function of $c$ both for our model and for the standard model. The minimum distance is higher in our example for $c<p_{x}-p_{m}$, and is the same otherwise. Intuitively, there are two effects which drive platforms of candidates apart. The first such effect, which is present both in our model and in the standard version, results from candidates trading off the cost of entry $c$ against the benefit from changing the outcome. Clearly, because of this effect, the minimum distance must rise with

\footnotetext{
${ }^{6}$ Examples of equilibria with more than two arbitrarily close candidates are available from the authors upon request.
} 


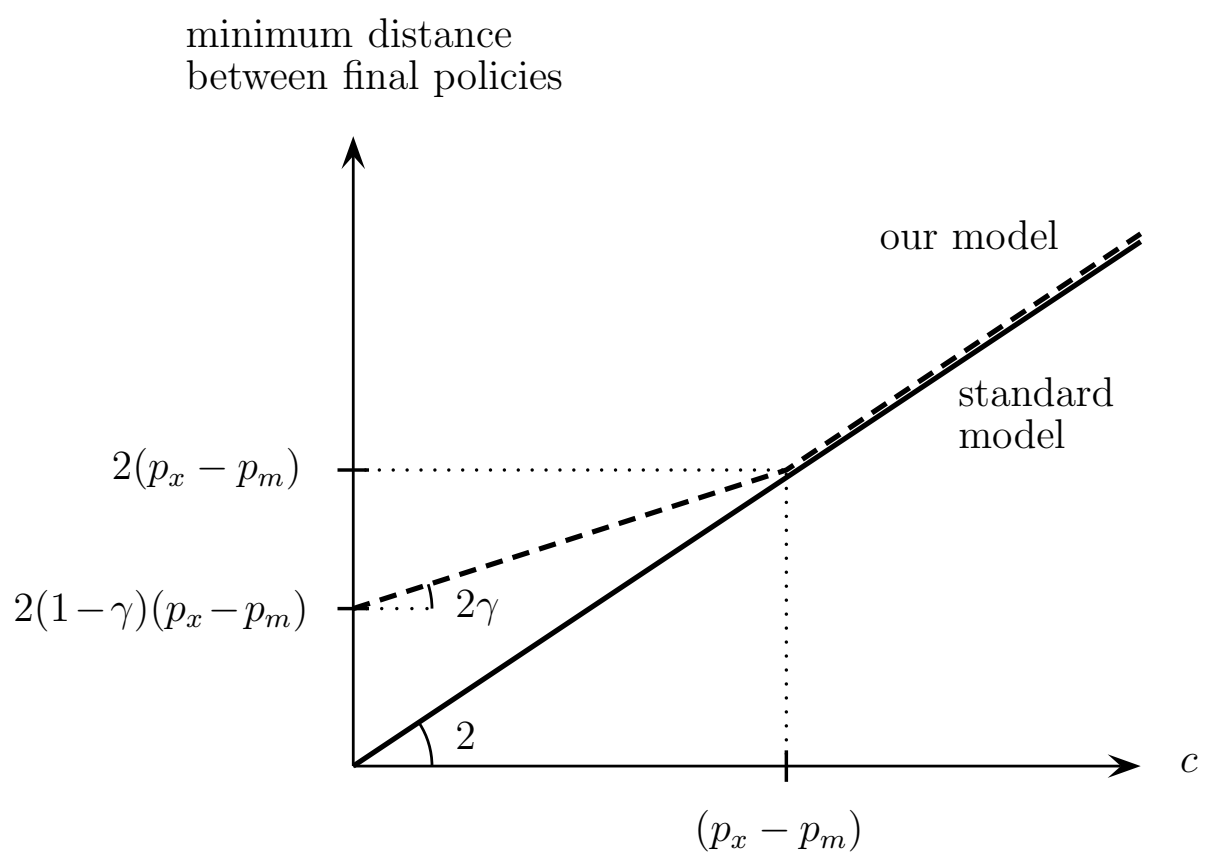

FIGURE 1 The minimum distance between final policies

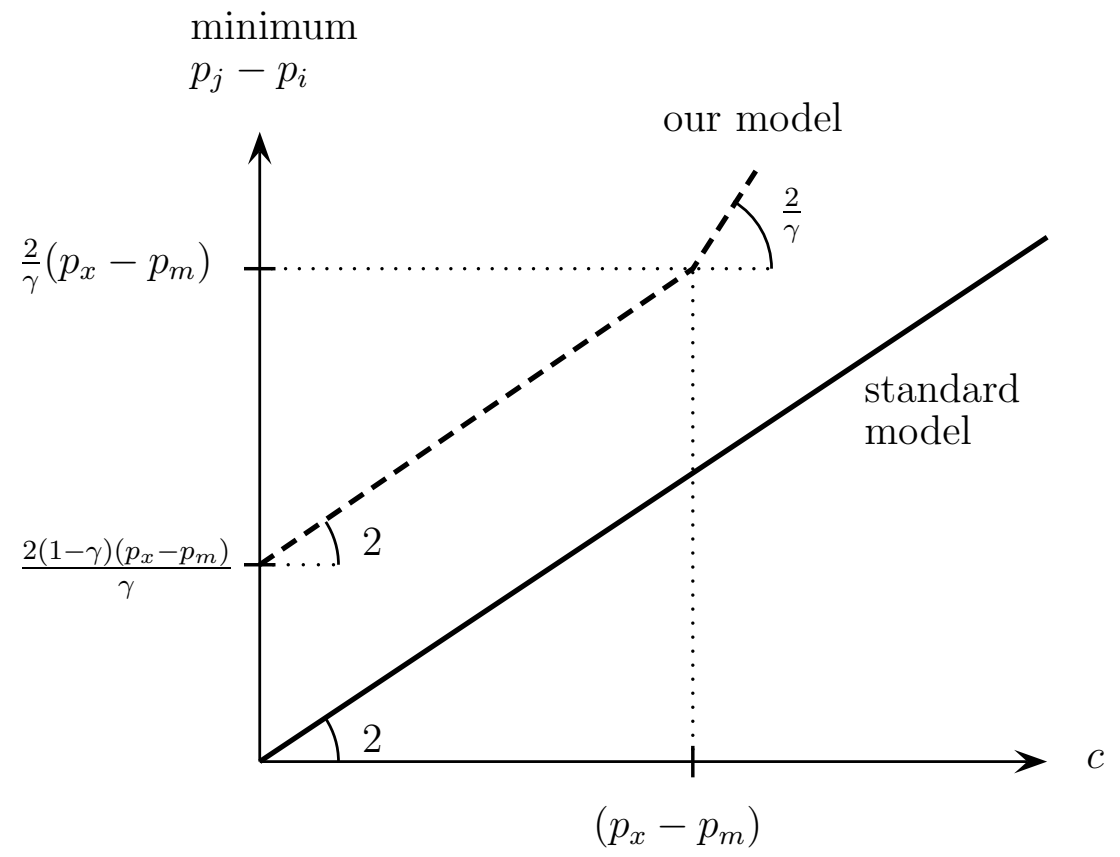

FIGURE 2 The minimum distance between ideal policies 
increases in $c$. The second effect is the strategic consideration arising from incomplete policy control: A candidate might want to lose against a candidate with a similar ideal point but who will achieve a better compromise with the outside power. ${ }^{7}$

When the entry cost is low compared to the ideological difference between the external power and the median voter $\left(c<p_{x}-p_{m}\right)$, both effects together determine the minimum distance of ideal points. However, as the above argument shows, when the entry cost is high $\left(c \geq p_{x}-p_{m}\right)$, an equilibrium where both candidates and the median voter prefer policies on the same side of $p_{x}$ is ruled out. That is, entry cost considerations alone drive possible ideal points so far away from each other that the strategic effect cannot operate any longer. In that sense, in terms of final policies, the model with imperfect policy control behaves like the standard model once the cost of entry exceeds the threshold $p_{x}-p_{m}$.

A further remark about Figure 1 is that as $c$ goes to 0, the minimum distance between final policies goes to $2(1-\gamma)\left(p_{x}-p_{m}\right)$ in our example. Notice that this is the double of the minimum distance in the general case ${ }^{8}$. The reason is that we assume the symmetry of the utility function around the ideal policy point for this example. In the general case, we only assumed singlepeakedness.

The graph in Figure 2 depicts the minimum distance between candidates' ideal policies as a function of $c$ both for our model and for the standard model. At $c=0$, the distance between ideal policies exceeds the distance between final policies by the factor $1 / \gamma$, which reflects the fact that the impact of candidates on final policies is mitigated by imperfect policy control. Furthermore, we see that the minimum distance is always higher in our model compared to the standard model. Even for entry cost beyond $p_{x}-p_{m}$, where the minimum distances between final policies in both models coincide, ideal policies still must be farther apart in our model than in the standard model because of the mitigated impact of candidates on final policies.

We further note that in this example, the two candidates' ideal policies must be situated on different sides of the median voter: Given that each citizen shares the same utility function except the ideal point and that the median voter is indifferent between the two final policies, every citizen to the left of the median prefers $p_{i x}$ over $p_{j x}$. Hence, the right-wing candidate $j$ 's ideal policy should be to the right of the median's since otherwise she would prefer her opponent's final policy and not enter the race. In contrast, in the general case we cannot rule out an equilibrium where both candidates are to the left of the median voter since citizens can have different types of utility functions.

To summarize, both figures illustrate the importance of the divergence of preferences between the outside power and the median voter. As $p_{x}$ moves farther to the right of $p_{m}$, the range of entry cost parameters where the model with incomplete policy control behaves differently from the standard model increases. Conversely, in the limit case where $p_{x} \rightarrow p_{m}$, the dotted and solid lines in Figure 1 coincide, so that incomplete policy control does not affect the minimum distance between final policies. Figure 2 shows that, in the limit case, the minimum distance between candidates' ideal policies is still larger than in the standard model, because candidates still cannot

\footnotetext{
${ }^{7}$ See the discussion after Corollary 1.

${ }^{8}$ Strictly speaking, in the general case, we do not fully characterize an equilibrium, rather we make use of necessary conditions in order to find out the minimum distance between final policies. However, the analysis of the general case can be turned into an equilibrium characterization by complementing it by the appropriate assumptions as in Besley and Coate (1997), Proposition 3. Hence, there indeed exist equilibria in the general case in which the distance between final policies is just larger than the minimum distance as given by Corollary 1, i.e. $(1-\gamma)\left(p_{x}-p_{m}\right)$.
} 
implement their preferred policies when $\gamma<1$.

When it comes to one-candidate equilibria, the first necessary condition is that the candidate prefers to run rather than not run. Moreover, whether a citizen running unopposed is an equilibrium depends on other citizens' positions since an equilibrium requires that there does not exist any other citizen who finds profitable to run as a candidate. Hence, the distribution of citizens' ideal positions determines the necessary condition to rule out entry of a second candidate. ${ }^{9}$ In the following Proposition 5, we characterize one-candidate equilibria which remain equilibria for all distributions of ideal positions for specified values of $c$, not necessarily small. In other words, we restrict our analysis to sufficient conditions. There may well be other equilibria for some distributions of ideal positions, but, if the conditions given in the proposition hold, then an equilibrium is robust against arbitrary changes in the location of ideal policies of all voters except the candidate and the median.

Since we are interested in the working of strategic delegation, a natural reference point is the policy $p^{*}$, defined by $\gamma p^{*}+(1-\gamma) p_{x}=p_{m}$, which will result in $p_{m}$ as the final policy. In the proposition, this policy features prominently.

PROPOSITION 5. Let any set of voters $N$ with ideal policies $\left(p_{1}, \ldots, p_{m}, \ldots, p_{n}\right)$ be given, and assume that utility functions are given by $u_{i}(p)=-\left|p-p_{i}\right|$ for all $i \in N$. Then there exists a one-candidate equilibrium in which citizen $i$ runs unopposed if
1. $c \leq \frac{2}{3}\left(p_{x}-p_{m}\right)$ and $p_{i} \in\left[2 p^{*}-p_{m}-\frac{c}{2}, p^{*}+\frac{c}{2 \gamma}\right]$ or
2. $\frac{2}{3}\left(p_{x}-p_{m}\right)<c \leq 2\left(p_{x}-p_{m}\right)$ and $p_{i} \in\left[2 p^{*}-p_{m}-\frac{c}{2}, p_{x}-\frac{c}{\gamma}\right]$.

Proof. The proof of Proposition 5 involves lengthy case distinctions and is therefore omitted. ${ }^{10}$

If there is a citizen whose ideal policy is $p^{*}$, this citizen is included in the intervals of both cases of the proposition. This proposition confirms that if there is a citizen who gives the median voter's most preferred policy as a final outcome, this citizen running unopposed constitutes a onecandidate equilibrium (unless the cost of entry is so high that this citizen prefers not to run). Hence, in the context of one-candidate equilibria, it is possible that the median voter offsets the influence of the external power through strategic delegation.

However, even for an arbitrarily small cost of entry, there remain other equilibria in which the candidate is not the one preferred by the median voter. For sufficiently low values of $c$, case 1. of Proposition 5 applies, which corresponds to the set of implemented policies: $p_{i x} \in\left[p_{m}-\right.$ $\left.(1-\gamma)\left(p_{x}-p_{m}\right)-\frac{c \gamma}{2}, p_{m}+\frac{c}{2}\right]$. By taking the limit of this set with respect to $c \rightarrow 0$, we see that the set of implemented policies converges to the interval $\left[p_{m}-(1-\gamma)\left(p_{x}-p_{m}\right), p_{m}\right]$. Therefore, even for arbitrarily small costs of entry, it is possible to sustain policies to the left of $p_{m}$ as equilibrium policies in our model. This is impossible in the standard citizen-candidate framework, in which there exists a unique one-candidate equilibrium for sufficiently small cost of entry, and the candidate is the median voter in this equilibrium.

\footnotetext{
${ }^{9}$ In the case of two-candidate equilibria, entry of a third candidate is ruled out by strategic voting of citizens who do not consider her as a serious contender and do not vote for her consequently. Hence, the distribution of ideal positions of other citizens does not matter. However, this argument does not apply for one-candidate equilibria since entry of a second candidate cannot trigger such a strategic voting.

${ }^{10}$ The detailed proof is available from the authors upon request.
} 
We also see that the corresponding sets of implemented policies, $p_{i x} \in\left[p_{m}-(1-\gamma)\left(p_{x}-\right.\right.$ $\left.\left.p_{m}\right)-\frac{c \gamma}{2}, p_{m}+\frac{c}{2}\right]$ for case 1. and $p_{i x} \in\left[p_{m}-(1-\gamma)\left(p_{x}-p_{m}\right)-\frac{c \gamma}{2}, p_{x}-c\right]$ for case 2., become larger as $1-\gamma$ increases. Hence, the imperfect policy control enlarges the sets of final policies in one-candidate equilibria as given by Proposition 5.

To summarize the discussion, on the one hand, we see that the median voter's preferred outcome is an equilibrium, so that strategic delegation may work. On the other hand, there is a whole interval of ideal policy positions where equilibrium candidates may be located, some of which yield final policies far away from the ideal point of the median voter. Moreover, for small cost of entry, some of these outcomes could not occur in a one-candidate equilibrium of the standard model, because the median voter would successfully enter the race against any candidate implementing a policy she strongly dislikes.

\section{Conclusion}

This paper extends the well-known citizen-candidate model by introducing some unelected entity such as a bureaucracy or foreign influences. It is assumed that the final policy arises from a compromise between the elected government and this outside power. We show that this extended model is structurally different from the standard model with perfect policy control by examining equilibria with one or two candidates running for office. The latter kind of equilibria necessarily display a divergence of ideal policies between candidates, and of final policies implemented from the preferences of the median, even if the cost of being a candidate is very small. In the same vein, a multiplicity of one-candidate equilibria, some of which lead to policy outcomes substantially different from the median voter's preferred policy, may exist even with negligible cost of entry. Thus, while the median voter result provides a useful benchmark in many political economic analyses, it may be misleading if an outside influence is relevant.

Politically, our results show that the influence exerted by international institutions such as the European Union or the IMF is likely to create policy divergence in the countries concerned, and to drive major political positions away from the center. Thus, the paper contributes to explaining why extreme political positions tend to become popular in countries which are subject to economic or other policy programs conceived by such institutions. Now in the model presented here, electing a government with such a position plays a useful role for the country concerned since it partially undoes the outside influence, and, thus, the model creates some understanding for voters' decision to elect extremist parties. In a wider perspective, however, such parties are generally seen critical because of their policy stance in areas unrelated to the interaction with foreign institutions. If such concerns are important, we think that our results have a normative implication for international institutions and the economic policy programs they promote. Specifically, while our simple model is obviously not meant to provide a comprehensive analysis of the complex economic and political situation in Greece or any other country hit by the Euro crisis, we think that in one respect, our analysis carries a lesson for European Union politics: Imposing policy on a country undermines centrist political positions in that country. Thus, whatever the economic merit of the reforms

required, we recommend that the European Union thinks also about the political consequences of imposing such measures from outside. 


\section{Appendix}

Proof of Proposition 2. Since $u_{i^{*}}\left(p_{i^{*} x}\right)-u_{i^{*}}\left(p_{x}\right) \geq c, i^{*}$ has an incentive to run. Moreover, if $i^{*}$ runs for office, no candidate $j \notin N_{m}^{*}$ can win against her since the median strictly prefers $i^{*}$ and is decisive by assumption A1. A candidate $j \in N_{m}^{*}$ with the same ideal point $p_{j}=p_{i^{*}}$ implements the same policy and hence cannot gain from being a candidate against $i^{*}$. Finally, if there is a candidate $j \in N_{m}^{*}$ with $p_{j} \neq p_{i^{*}}$, it must hold $p_{i^{*}}<p_{j}<p_{m}$, and from singlepeakedness, $p_{i^{*} x}<p_{m}<p_{j x}$. Moreover, $u_{m}\left(p_{i^{*} x}\right)=u_{m}\left(p_{j x}\right)$ since both candidates are optimal for the median. Therefore, A1 implies that candidate $j$, who is to the left of the median, prefers the more left-wing of both policies, that is, $u_{j}\left(p_{i^{*} x}\right)>u_{j}\left(p_{j x}\right)$. Therefore, citizen $j$ prefers the outcome which candidate $i^{*}$ implements to the one she can achieve herself and thus is better off not running.

Proof of Proposition 3. For the if part of the claim, observe first that for a candidate with ideal policy $p_{i^{*}}$, the claim is already proven in Proposition 2. Let therefore $i$ be an arbitrary citizen with ideal policy $p_{i} \in\left[p_{l} ; p_{i^{*}}\right)$. From $p_{i}<p_{i^{*}}$ it follows that $p_{i x}<p_{i^{*} x} \leq p_{m x}<p_{x}$, which implies with single-peakedness that $u_{i}\left(p_{i x}\right)>u_{i}\left(p_{x}\right)$. Thus, for low enough $c$, citizen $i$ chooses to be a candidate if no one else runs.

We show that any other citizen $j$ either loses the election if she runs against $i$ or she does not want to oppose $i$ because she prefers $p_{i x}$ to $p_{j x}$. We distinguish cases.

Case 1: $p_{j}<p_{i}$. If $p_{j}<p_{i}$ then $p_{j x}<p_{i x}$. In addition, note that because $p_{i^{*} x}$ is the policy most preferred by the median voter the policy $p_{i x}$ implemented by citizen $i$ cannot be in between $p_{m}$ and $p_{i^{*} x}$. If this were the case then, by single-peakedness, $m$ would prefer $p_{i x}$ to $p_{i^{*} x}$ which would invalidate the definition of $i^{*}$. As a result, $p_{i x}<\min \left\{p_{m}, p_{i^{*} x}\right\}$. Therefore, we have the following inequalities: $p_{j x}<p_{i x}<p_{m}$. By single-peakedness, the median strictly prefers $i$ over $j$, and from A1, $i$ wins over $j$.

Case 2: $p_{i} \leq p_{j} \leq p_{i x}$. In this case, if $p_{i}=p_{j}, j$ will not change the outcome, and hence it is optimal for $j$ not to enter the race against $i$. If $p_{i}<p_{j}$, we have $p_{i x}<p_{j x}$, and overall, $p_{j} \leq p_{i x}<p_{j x}$. Therefore, by single-peakedness $j$ strictly prefers $p_{i x}$ to $p_{j x}$ and so she prefers not to run against $i$.

Case 3: $p_{i x}<p_{j} \leq p_{m}$. By definition of $l$, it must hold $u_{m}\left(p_{l x}\right) \geq u_{m}\left(p_{m x}\right)$. Hence, from Assumption A2 and since $j$ satisfies $p_{l} \leq p_{j} \leq p_{m}$, we must also have $u_{j}\left(p_{l x}\right) \geq u_{j}\left(p_{j x}\right)$. Moreover, since $p_{l x} \leq p_{i x}<p_{j}$, by single-peakedness, $u_{j}\left(p_{i x}\right) \geq u_{j}\left(p_{l x}\right)$. Combining these inequalities we obtain $u_{j}\left(p_{i x}\right) \geq u_{j}\left(p_{l x}\right) \geq u_{j}\left(p_{j x}\right)$. Therefore, $j$ weakly prefers $p_{i x}$ to $p_{j x}$ and so it is optimal for her not to run against $i$.

Case 4: $p_{j}>p_{m}$. This case implies that $p_{m x}<p_{j x}$, so $u_{m}\left(p_{m x}\right)>u_{m}\left(p_{j x}\right)$. By the definition of $p_{l}$, from $p_{l} \leq p_{i}$, and from single-peakedness, $u_{m}\left(p_{i x}\right) \geq u_{m}\left(p_{l x}\right)$ and $u_{m}\left(p_{l x}\right) \geq u_{m}\left(p_{m x}\right)$. Overall, $u_{m}\left(p_{i x}\right)>u_{m}\left(p_{j x}\right)$, so that $j$ loses against $i$ if she runs, by assumption A1.

For the only-if part we show that for any citizen $i$ whose ideal policy does not belong to $\left[p_{l}, p_{i^{*}}\right]$ there exists a citizen $j$ who wants to run against $i$ and has a chance of winning the election. 
Case 1: $p_{i}<p_{l}$. By the definition of $p_{l}$, we have that $u_{m}\left(p_{m x}\right)>u_{m}\left(p_{i x}\right)$, so that $m$ wants to run against $i$. Moreover, by assumption A1, $m$ wins the election.

Case 2: $p_{i}>p_{i^{*}}$ and $i \notin N_{m}^{*}$. Then $p_{i^{*}}<p_{i^{*} x}<p_{i x}$. Therefore, by single-peakedness, $u_{i^{*}}\left(p_{i^{*} x}\right)>u_{i^{*}}\left(p_{i x}\right)$, so $i^{*}$ wants to run against $i$. Since $i^{*} \in N_{m}^{*}$ but $i \notin N_{m}^{*}$, the median strictly prefers $i^{*}$ to $i$, and hence $i^{*}$ wins in a race against $i$ by assumption A1.

Case 3: $p_{i}>p_{i^{*}}$ and $i \in N_{m}^{*}$. Then $u_{m}\left(p_{i^{*} x}\right)=u_{m}\left(p_{i x}\right)$, and hence, by assumption $\mathrm{A} 1$, an election with $i^{*}$ and $i$ as candidates results in a tie. Therefore, entering the race against $i$ yields a benefit of $\frac{1}{2}\left[u_{i^{*}}\left(p_{i^{*} x}\right)-u_{i^{*}}\left(p_{i x}\right)\right]-c$ to citizen $i^{*}$. Since the median is indifferent between $p_{i^{*} x}$ and $p_{i x}$ and from $p_{i}>p_{i^{*}}$, we know that $p_{i^{*}}<p_{i^{*} x}<p_{m}<p_{i x}$. Hence, by single-peakedness, $u_{i^{*}}\left(p_{i^{*} x}\right)>u_{i^{*}}\left(p_{i x}\right)$. Consequently, for sufficiently low cost $c, i^{*}$ 's benefit from being a candidate is strictly positive, so that $i$ will not run unopposed.

QED

Proof of Proposition 4. Condition (b), which states that both candidates' gain from running is higher than the cost of entry, requires

$$
\begin{aligned}
& \frac{1}{2}\left[u_{i}\left(p_{i x}\right)-u_{i}\left(p_{j x}\right)\right] \geq c \\
\text { and } \quad \frac{1}{2}\left[u_{j}\left(p_{j x}\right)-u_{j}\left(p_{i x}\right)\right] & \geq c
\end{aligned}
$$

Condition (a), which requires that the median voter is indifferent between the two candidates, leads to $-\left|p_{i x}-p_{m}\right|=-\left|p_{j x}-p_{m}\right|$. In equilibrium, $p_{i x} \neq p_{j x}$, since otherwise condition (b) cannot hold. Hence, condition (a) becomes $p_{m}-p_{i x}=p_{j x}-p_{m}$, or equivalently,

$$
\frac{p_{i x}+p_{j x}}{2}=p_{m}
$$

We first analyze the case in which $p_{j}<p_{x}$. We know already from the general case (see the proof of Proposition 1) that we must have $p_{j}>p_{i x}$, since otherwise $j$ would have no interest in running. Then, conditions (A1) and (A2) translate to $-\left(p_{i x}-p_{i}\right)+\left(p_{j x}-p_{i}\right) \geq 2 c$ and $-\left(p_{j x}-p_{j}\right)+\left(p_{j}-p_{i x}\right) \geq 2 c$ or equivalently to

$$
\text { and } \begin{aligned}
p_{j x}-p_{i x} & \geq 2 c \\
2 p_{j}-p_{j x}-p_{i x} & \geq 2 c
\end{aligned}
$$

Since $p_{j x}>p_{j}$, we can see that if condition (A5) is satisfied, then condition (A4) is also satisfied. Using equation (A3), we can write condition (A5) as $p_{j} \geq p_{m}+c$.

Notice that the last condition is consistent with the assumption $p_{j}<p_{x}$ only if $c<p_{x}-p_{m}$. In other words, if $c \geq p_{x}-p_{m}$, there cannot be a two-candidate equilibrium with $p_{j}<p_{x}$. We also have $p_{j x}=\gamma p_{j}+(1-\gamma) p_{x} \geq \gamma\left(p_{m}+c\right)+(1-\gamma) p_{x}$ which leads to $p_{j x}-p_{m} \geq(1-\gamma)\left(p_{x}-p_{m}\right)+\gamma c$. Since $p_{m}-p_{i x}=p_{j x}-p_{m}$, we conclude that $p_{j x}-p_{i x} \geq 2(1-\gamma)\left(p_{x}-p_{m}\right)+2 \gamma c$, and consequently $p_{j}-p_{i} \geq 2[(1-\gamma) / \gamma]\left(p_{x}-p_{m}\right)+2 c$.

We now analyze the case in which $p_{j} \geq p_{x}$. In this case, conditions (A1) and (A2) translate to $-\left(p_{i x}-p_{i}\right)+\left(p_{j x}-p_{i}\right) \geq 2 c$ and $-\left(p_{j}-p_{j x}\right)+\left(p_{j}-p_{i x}\right) \geq 2 c$ which both lead to $p_{j x}-p_{i x} \geq 2 c$. 
Since we assumed $p_{j} \geq p_{x}$, we also have $p_{j x}-p_{i x}=2\left(p_{j x}-p_{m}\right) \geq 2\left(p_{x}-p_{m}\right)$. Hence, $p_{j x}-p_{i x} \geq \max \left\{2 c, 2\left(p_{x}-p_{m}\right)\right\}$ and consequently $p_{j}-p_{i} \geq \max \left\{2 c / \gamma, 2\left(p_{x}-p_{m}\right) / \gamma\right\}$.

Integrating the analysis of the two cases, we see that, if $c \geq p_{x}-p_{m}$, any two-candidate equilibrium is such that $p_{j} \geq p_{x}$, and the necessary condition for the minimum distance between final policies is given by $p_{j x}-p_{i x} \geq 2 c$. If $c<p_{x}-p_{m}$, the necessary condition for the minimum distance is given by $p_{j x}-p_{i x} \geq 2(1-\gamma)\left(p_{x}-p_{m}\right)+2 \gamma c$ for equilibria with $p_{j}<p_{x}$, and by $p_{j x}-p_{i x} \geq 2\left(p_{x}-p_{m}\right)$ for equilibria with $p_{j} \geq p_{x}$. The necessary condition for equilibria with $p_{j}<p_{x}$ is less restrictive. Hence, we conclude that if $c<p_{x}-p_{m}$, the necessary condition for the minimum distance is given by $p_{j x}-p_{i x} \geq 2(1-\gamma)\left(p_{x}-p_{m}\right)+2 \gamma c$.

QED

\section{References}

Besley, T., and S. Coate (1997) "An economic model of representative democracy." The Quarterly Journal of Economics 112, 85-114

Besley, T., and S. Coate (2001) "Lobbying and welfare in a representative democracy." Review of Economic Studies 68, 67-82

Brusco, S., and J. Roy (2011) "Aggregate uncertainty in the citizen candidate model yields extremist parties." Social Choice and Welfare 36, 83-104

Chambers, C. (2007) "Citizen-candidates, lobbies, and strategic campaigning." Economic Theory 33, 285-309

Chattopadhyay, R., and E. Duflo (2004) "Women as policy makers: Evidence from a randomized policy experiment in India." Econometrica 72, 1409-1443

Fatum, R. (2006) "One monetary policy and 18 central bankers: The European monetary policy as a game of strategic delegation." Journal of Monetary Economics 53, 659-669

Hamlin, A., and M. Hjortlund (2000) "Proportional representation with citizen candidates." Public Choice 103, 205-230

Harstad, B. (2010) "Strategic delegation and voting rules." Journal of Public Economics 94, 102-113

Jennings, C., and H. Roelfsema (2008) "Civil conflict, federalism and strategic delegation of leadership." Journal of Peace Research 45, 557-573

Niskanen, W. A. (1971) Bureaucracy and Representative Government (Chicago: Aldine-Atherton)

Osborne, M., and A. Slivinsky (1996) "A model of political competition with citizen-candidates." The Quarterly Journal of Economics 111, 65-96

Persson, T., and G. Tabellini (1992) “The politics of 1992: Fiscal policy and European integration." The Review of Economic Studies 59, 689-701

Persson, T., and G. Tabellini (1994) "Representative democracy and capital taxation.” Journal of Public Economics 55, 53-70

Persson, T., and G. Tabellini (2002) Political Economics: Explaining Economic Policy (Cambridge, Mass.: The MIT Press)

Roelfsema, H. (2007) "Strategic delegation of environmental policy making." Journal of Environmental Economics and Management 53, 270-275 